\title{
An Intelligence Fault Tolerance Agent for Multimedia CSCW based on Situation- Awareness
}

\author{
Eung-Nam Ko \\ Division of Information $\mathcal{E}$ Communication, Baekseok University
}

Korea

\section{Introduction}

CSCW (Computer Supported Cooperative Work) is the study of how people use technology, with relation to hardware and software, to work together in shared time and space. CSCW began as an effort by technologists to learn from anyone whom could help them better understand group activity and how one could use technology to support people in their work (Grudin J, 1994). There are two dimensions to CSCW-space and time. Figure 1 shows examples relevant to this study in the usual quadrants (J Lama et al, 2006). In this study, we describe video conferencing in the 3 rd quadrant.

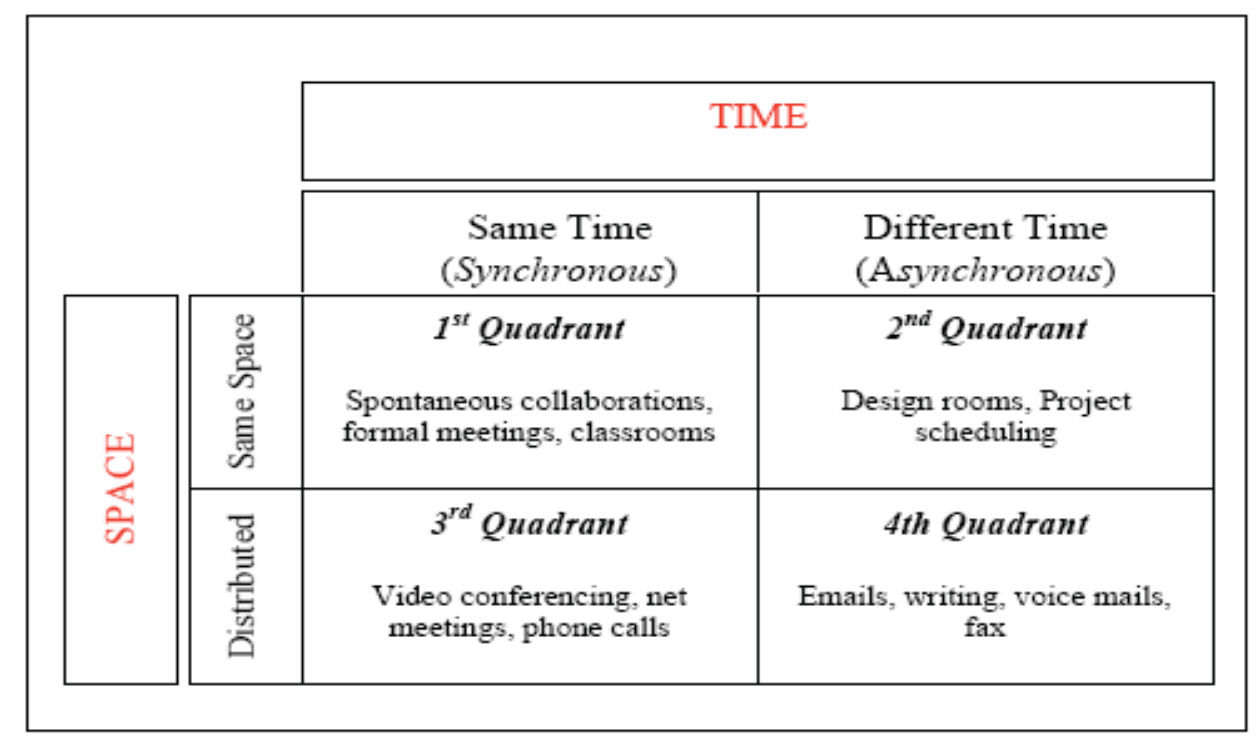

Fig. 1. CSCW quadrants 
Distance education is planned learning that normally occurs in a difference place from teaching and as a result requires special techniques of course design, special instructional techniques, special methods of communication by electronic and other technology, as well as special organizational and administrative arrangements. It presents a general systems model that describes the main component processes and elements of a distance education institution, program, unit, consortium, or course (Michael G.Moore et al, 1996).

A system includes the subsystems of knowledge sources, design, delivery, interaction, learning, and management. The more integrated these are in practice, the greater will be the effectiveness of the distance education organization (Jae Young Ahn et al, 1996).

The implementation of interactive multimedia distance education system can be recognized as a diversification of videoconferencing system which first appeared in the 1980's. Early implementations of videoconferencing systems were circuit-based systems relying on dedicated video devices, telephone networks or leased lines. After the early 1990's, the major basis of videoconferencing system moved to packet-based systems which operate on computer network (Francois Fluckiger, 1995; C.W.Loftus et al, 1995).

However, since this new education system must be developed in a way that combines various field of technologies, including group communication and distributed multimedia processing which are the basis of packet based videoconferencing systems, integrated service functions such as middle ware are required to support it (ITU-T Recommendation, 1995).

The development of middleware is closely related to the evolution of ubiquitous computing began in the mid of 1970s, when the PC first brought computers closer people. With the advent of networking, personal computing evolved into distributed computing. With seamless access and World Wide Web, distributed computing marked a next step toward pervasive computing, and mobile computing emerged from the integration of cellular technology with the Web. The "anytime anywhere" goal of mobile computing is essentially a reactive approach to information access, and it prepare the way for pervasive computing's proactive "all the time everywhere" goal (Satyanarayanan, M , 2001; Saha, D, Mukherjee, A. 2003).

The requirement of distributed multimedia applications is the need for sophisticated quality of service(QoS) management. In most traditional computing environments, requests for a particular service are either met or ignored. In a multimedia system, however, the quality of the service achieved is central to the acceptability of the application. In terms of distributed multimedia systems, the most important categories for quality of service are a timeless, volume, and reliability (Gordon Blair, Jean-Bernard Stefani, 1997).

In this chapter of book, we propose a method for increasing reliability. The rest of this chapter of book is organized as follows. Section 2 describes context awareness. Section 3 denotes our approach. Section 4 evaluate and concludes the chapter.

\section{Related Works}

Distance education systems must be able to support real-time interaction, temporal/spatial synchronization and floor control for smooth interaction (Jae Y. Ahn et al, 1996; Gil C. Park et al, 1995).

In ubiquitous computing environment, application should be able to properly adapt itself according to its own context information coming from ubiquitous sensors (Younglok Lee et 
al, 2006). The recently many interest comes to collect in about the sensor network and it has environmental monitoring, a weather measurement, a military field and etc. specific goal and actively from many field, the researches are advanced (Man Seok Yang et al, 2009).

Context awareness (or context sensitivity) is an application software system's ability to sense and analyze context from various sources; it lets application software take different actions adaptively in different contexts (S. Yau, F. Karim et al, 2002). In a ubiquitous computing environment, computing anytime, anywhere, any devices, the concept of situation-aware middleware has played very important roles in matching user needs with available computing resources in transparent manner in dynamic environments (S. Yau, F. Karim et al, 2002). Although the situation-aware middleware provides powerful analysis of dynamically changing situations in the ubiquitous computing environment by synthesizing multiple contexts and users' actions, which need to be analyzed over a period of time, it is difficult to analyze Quality of Service (QoS) of situation-aware applications because the relationship between changes of situations and resources required to support the desired level of QoS is not clear.

The field of fault-tolerant computing has evolved over the past twenty-five years $(\mathrm{H}$. Zhang A et al, 1995). In spite of this current trend, however, study on fault- tolerance of application software has not actually been enough. Generally, fault-tolerance system can be classified as software techniques, hardware techniques and composite techniques (Victor P. Nelson and Bill D. Carroll). The tolerance of software faults is in most cases more difficult than dealing with hardware faults since most software-fault mechanisms are not well understood and do not lend themselves readily to "nice" techniques such as error coding (Dhiraj K. Pradhan, 1996).

Since the application needs of middleware services and computing environment (resources) keep changing as the application change, it is difficult to analyze: whether it is possible that Quality of Service (QoS) of requirements for error-detection recovery are met, and what QoS requirements for error-detection recovery have tradeoff relationships.

Thus, there is a great need for fault tolerance algorithm in situation-aware middleware to provide dependable services in ubiquitous computing in case of errors occurrence. Context awareness (or context sensitivity) is an application software system's ability to sense and analyze context from various sources; it lets application software take different actions adaptively in different contexts. With the rapid development of multimedia and network technology, more and more digital media is generated (T. Zhang et al, 1999). Although the situation-aware middleware provides powerful analysis of dynamically changing situations in the ubiquitous computing environment by synthesizing multiple contexts and users' actions, which need to be analyzed over a period of time, access control in using multimedia shared object causes a problem of the seam in the ubiquitous computing environment. It is difficult to avoid a problem of the seam in the ubiquitous computing environment for seamless services. There is a great need for situation-aware middleware to be able to predict whether all QoS requirements of the applications are satisfied and analyze tradeoff relationships among the QoS requirements, if all QoS requirements cannot be satisfied to determine a higher priority of QoS requirements.

This paper proposes a new model of intelligence fault tolerance agent for multimedia CSCW based on situation-aware ubiquitous computing. It is analyzing the window and attributes of the object, and based on this, a mechanism that offers a seamless multimedia view without interfering with access control is also suggested. Our FTA model is to present the 
relationship of missions, actions, QoS and resources. FTA model is used to detection and recover the QoS resource errors among actions.

\section{Our Approach}

\subsection{A Situation-Awareness Middleware: RCSM}

In this paper, we propose a new access control mechanism in situation-aware middleware. A conceptual architecture of situation-aware middleware based on Reconfigurable ContextSensitive Middleware (RCSM) is proposed in (Saha, D, Mukherjee, A., 2003). Figure 2 shows Architecture of RCSM.

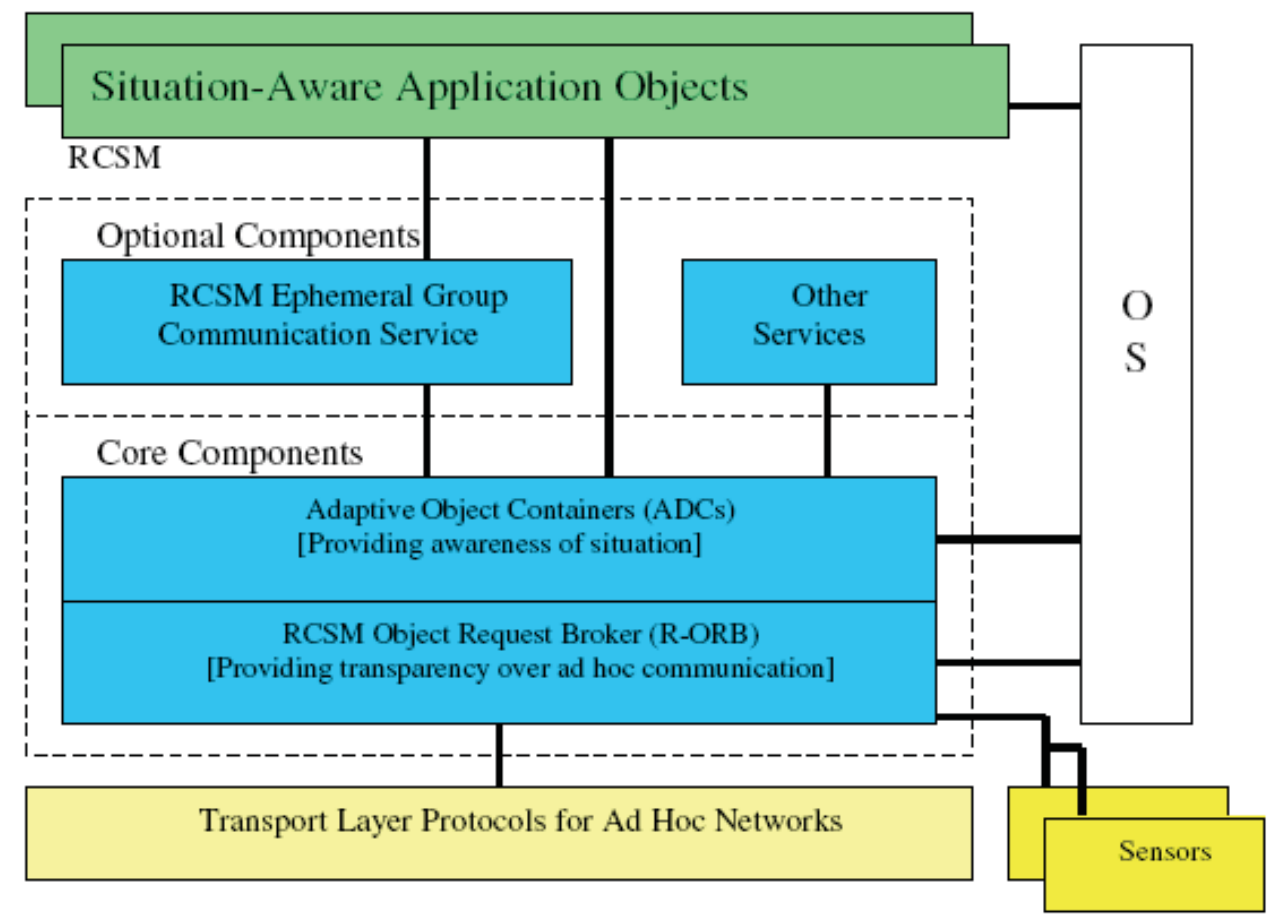

Fig. 2. Architecture of RCSM

All of RCSM's components are layered inside a device. The Object Request Broker of RCSM (R-ORB) assumes the availability of reliable transport protocols; one R-ORB per device is sufficient. The number of ADaptive object Containers (ADC) s depends on the number of context-sensitive objects in the device. ADCs periodically collect the necessary "raw context data" through the R-ORB, which in turn collects the data from sensors and the operating system. Initially, each ADC registers with the R-ORB to express its needs for contexts and to publish the corresponding context-sensitive interface. RCSM is called reconfigurable because it allows addition or deletion of individual ADCs during runtime (to manage new 
or existing context-sensitive application objects) without affecting other runtime operations inside RCSM (Saha, D, Mukherjee, A., 2003).

Ubiquitous applications require use of various contexts to adaptively communicate with each other across multiple network environments, such as mobile ad hoc networks, Internet, and mobile phone networks. However, existing context-aware techniques often become inadequate in these applications where combinations of multiple contexts and users' actions need to be analyzed over a period of time. Situation-awareness in application software is considered as a desirable property to overcome this limitation. In addition to being contextsensitive, situation-aware applications can respond to both current and historical relationships of specific contexts and device-actions. An example of situation-aware applications is a multimedia distance education system. The development of multimedia computers and communication techniques has made it possible for a mind to be transmitted from a teacher to a student in distance environment. However, it did not include access control support in the architecture of situation-aware middleware.

However, it did not include QoS support in the architecture. In this paper, we focus on how to represent QoS requirements in situation-aware middleware. In the next subsection, we will present a conceptual model for QoS requirements representation in situation-aware middleware.

\subsection{RCSM Optional Components: DOORAE}

Ubiquitous applications require use of various contexts to adaptively communicate with DOORAE agent layer includes many agents. They are AMA (Application Management Agent), IA (Intelligent Agent), SMA (Session Management Agent), ACA (Access Control Agent), MCA (Media Control Agent) , and FTA (Fault Tolerance Agent). The organization of DOORAE agent layer running on RCSM is shown in Figure 3.

AMA (Application Management Agent) consists of various subclass modules. These subclass modules provide the basic agent, while AMA supports a mixture of various basic services. AMA includes creation/deletion of shared video window and creation/deletion of shared window. For providing heterogeneous platforms with interoperability, it is necessary to share media data and to furnish awareness to the remote users involved in collaborative work. To solve the problem, we set the IA (Intelligent Agent) that modifies the transmitting packets by using TCP/ IP or UDP. Event messages including information about shared objects are bypassed among the homogeneous. SMA (Session Management Agent) controls the access to the whole session. This agent can be used in meeting, distance learning, playing games and development of any software. Session control also facilitates access and limits it to the whole session. ACA (Access Control Agent) controls the person who can talk, and the one who can change the information. The mechanism of floor control consists of brainstorming, priority, mediated, token-passing and time-out. MCA (Media Control Agent) support convenient application using DOORAE based on RCSM environment. Supplied services are the creation and deletion of the service object for media use, and media share between the remote users. This agent limits the service by hardware constraint. 


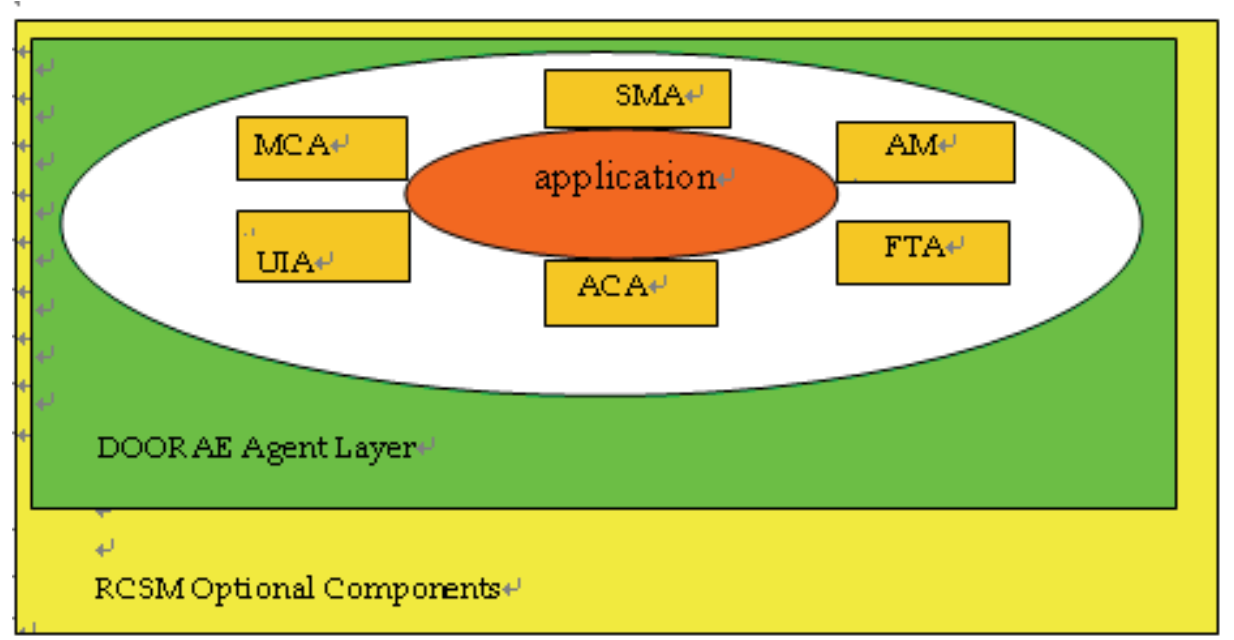

Fig. 3. The Organization of DOORAE

\subsection{FTA based on RCSM Optional Components}

FTA consists of EDA(Error Detection Agent) and ERA(Error Recovery Agent). EDA consists of ED(Error Detector), EC(Error Classifier), and EL(Error Learner). FTA is an agent which plays a role in detecting, classifying, and recovering errors. As shown in Figure 4, you can see the message flows in the organization of FTA.

ED is an agent which plays a role as an interface to interact among an application, EC, and EL. ED has functions which detect an error. ED informs EC of the results of detected errors. ED inspects applications by using process database function periodically to find an error. EC and EL deal with learning in reactive multi-agent systems. Generally, learning rules may be classified as supervised or unsupervised. In this paper, it uses a perception training. Hence, the training set consists of a set of input vectors, each with its desired target vector. Input vector components take on a continuous range of values; target vector components are binary valued (either zero or one). After training, the network accepts a set of continuous inputs and produces the desired binary valued outputs. KB has a registration information of creation of service handle and session manager handle by Daemon and GSM. EC can decide whether it is hardware error or software error based on learning rules by EL. In case of hardware error, it cannot be recoverable. In case of software error, it can be recoverable. This approach is based on the idea of comparing the expected error type which is generated by an EL with the actual error occurred from sites. 


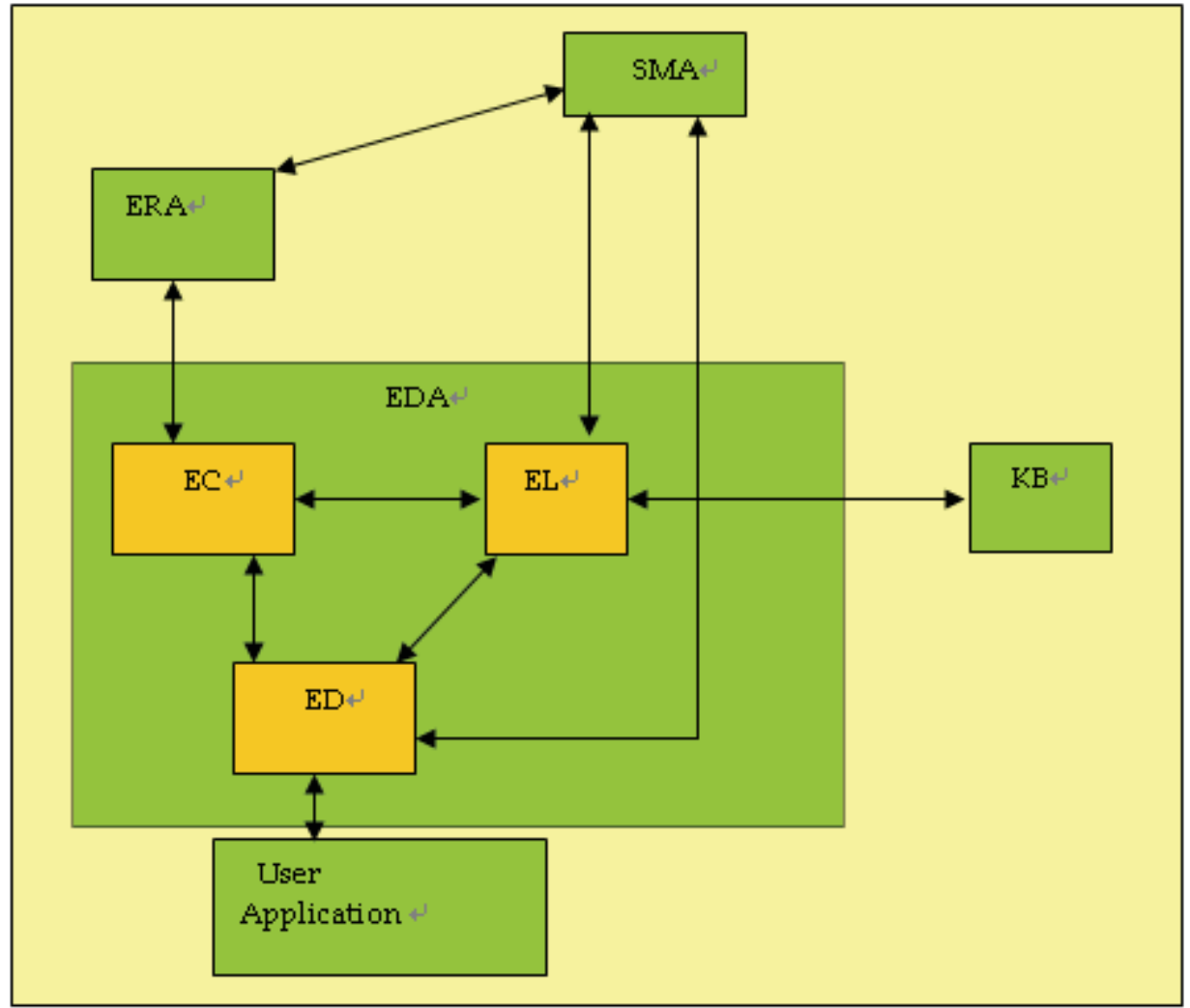

Fig. 4. The Organization of FTA based on RCSM Optional Components

Second, EC is an agent that plays a role as an interface to interact between ED for detection and ER for recovery such as Figure 5. EC and EL have functions which classify the type of errors by using learning rules. EC and EL deal with learning in reactive multi-agent systems. Generally learning rules may be classified as supervised or unsupervised or reinforcement learning. Reinforcement learning is similar to supervised learning, except it, instead of being provided with the concept output for each network input, the algorithm is only given a grade. The grade (or score) is a measure of the network performance over some sequence of inputs. This paper deals with Q-learning that is one of the reinforcement learning. Because EC and EL have not knowledge of error classification, it receives an acknowledge information which is necessary for fault diagnosis from PDB (Process Data Base). Hence the training set consists of a set of input vectors, each with its desired target vector. Input vector components take on a continuous range of values. Target vector components valued. After training, the network accepts a set of continuous inputs and produces EC and EL can decide whether it is hardware error or software error based on learning rules. 


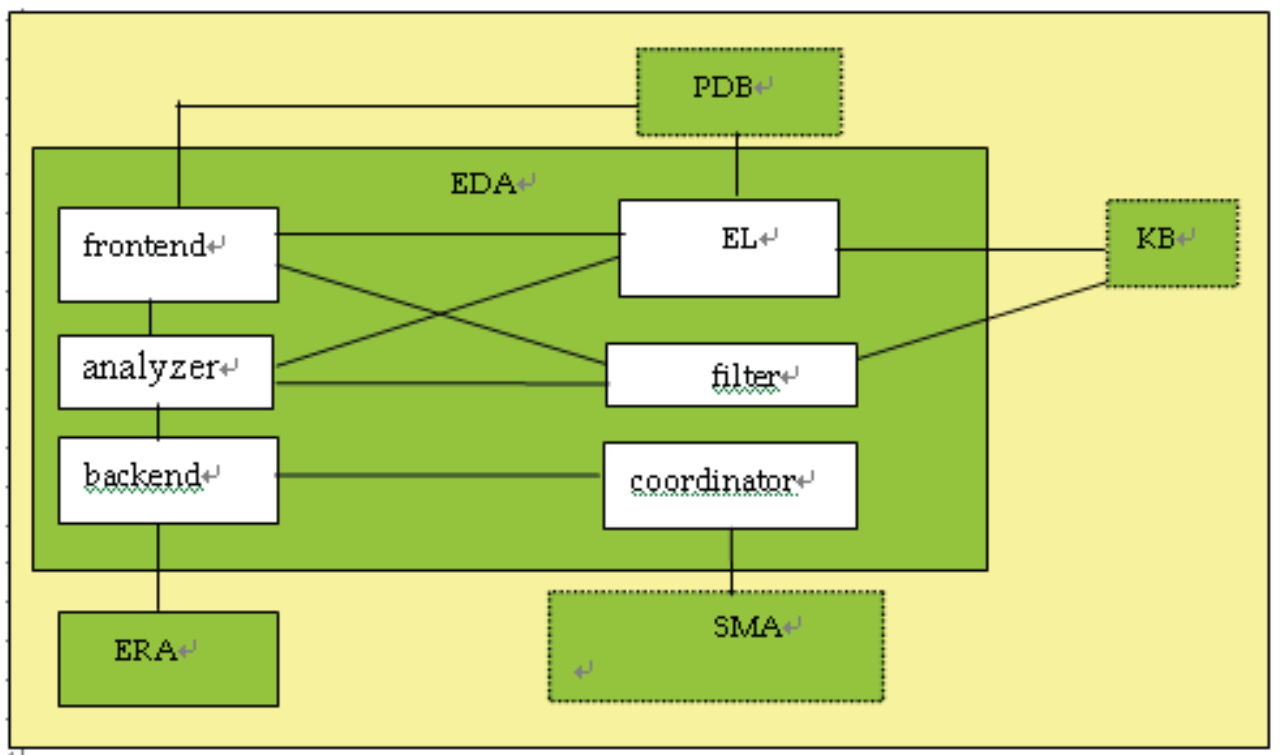

Fig. 5. The Organization of EC and EL

The scheme of classification mode is as follows. It is an ordered set. EC consists of frontend, backend, analyzer, coordinator, filter. Frontend has a function of playing a role in receiving error detection information from ED. Backend has a function of playing a role in receiving error recovery information from ERA. Coordinator informs SMA of the result. Analyzer has a function of classifying error's information that is received from frontend. Filter has a function of storing an error's history information in KB from error information that is classified by EL. EL has a function of classifying the type of errors by using learning rules with consideration of information from analyzer.

The sequence of Recovery's message flow can be shown in Figure 6. If an error is to be recovered, you can create sequences below. It creates a session with initial configuration information. It requests port ids for audio/video servers to build-up a Local Session Manager. It assigns port ids for audio/video servers of an application. It invites to the session and build-up a session instance monitor. It sends invited messages to start build-up of session instance monitor. It builds up Session Instance Monitor using the configuration information from LSM. It sends joint message to the Local Session Manager. It sends session information to Global Session Manager for set-up of GSM table. It begins a session. It exchanges message or command between LSM and PSM and media data between media server based on interpretation of message handler. 


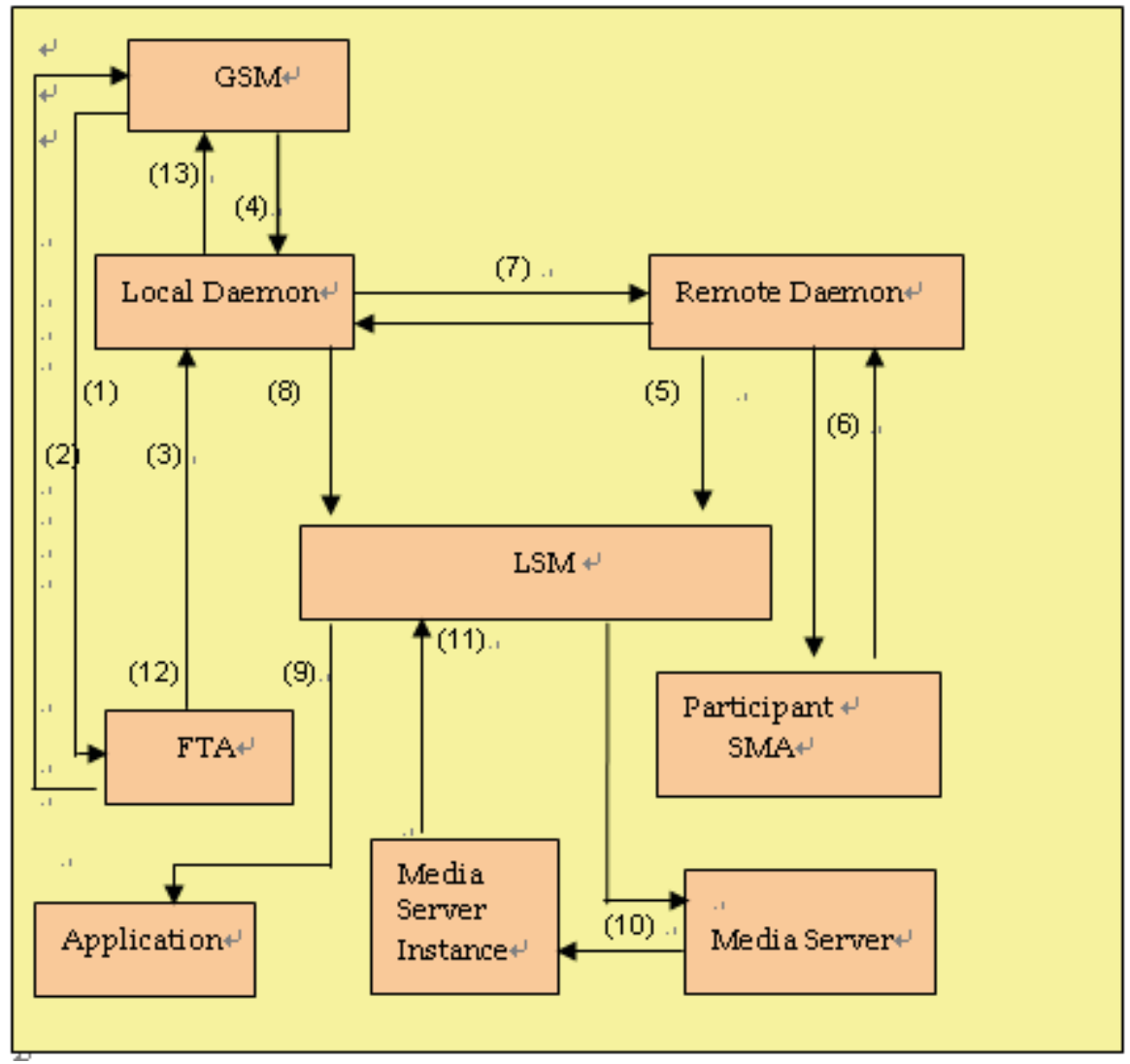

Fig. 6. Message Flow for Recovery

First it is decided whether it is hardware error or software error. In case of software error, it can be recoverable. If an error is to be recoverable, you can create sequences below. FTA requests to GSM session information. -GSM give response FTA session information. FTA requests to Daemon for recovery. Daemon announce to Remote-Daemon for recovery. Remote-Daemon announce to Participant Session Manager for recovery. Remote-Daemon receives an acknowledgement for recovery packet. Daemon receives an acknowledgement for recovery packet. Daemon creates Local Session Manager. Local Session Manager create Media server. Media server create Media server Instance. Media server Instance makes an acknowledgement to Local Session Manager. LSM creates application. Daemon informs SAGSM of an information for recovery. The strong point of this system is to detect and recovered autonomously in case that the session's process come to an end from software error.

Our proposed FTA model aims at supporting adaptive QoS requirements defined in application-level Missions described by a set of Actions of objects by reserving, allocating, and reallocating necessary Resources given dynamically changing situations. A high-level FTA conceptual architecture to support adaptive QoS requirements is shown in Figure 7. 


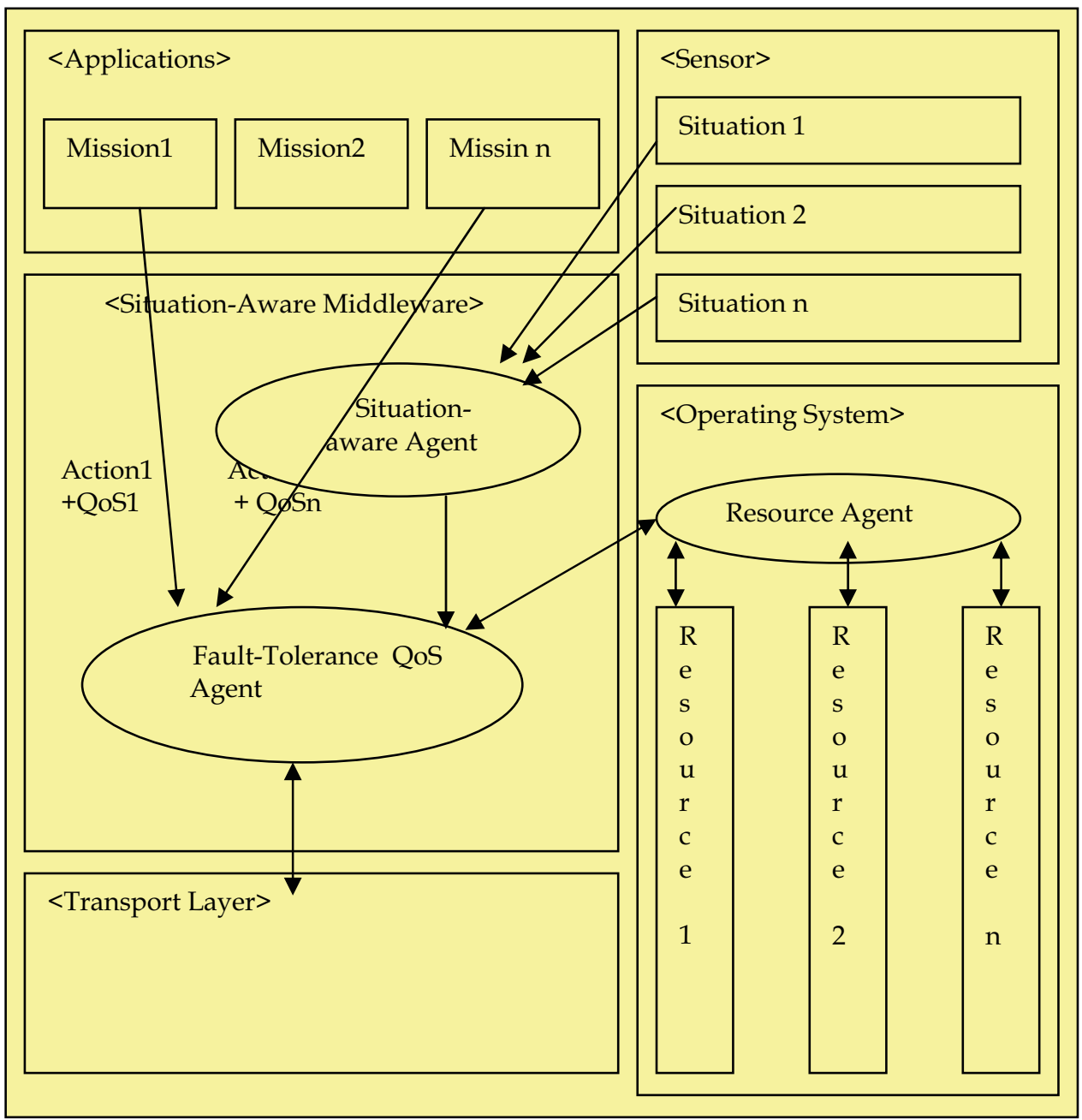

Fig. 7. Message Flow for QoS Architecture of RCSM

Situation-aware Manager, Resource Manager, and QoS Management Agent are the main components shown in Situation-Aware Middleware. Applications request to execute a set of missions to Situation-aware Middleware with various QoS requirements. A Situation-aware Manager analyzes and synthesizes context information (e.g., location, time, devices, temperature, pressure, etc.) captured by sensors over a period of time, and drives a situation. A Resource Manager simultaneously analyzes resource availability by dividing requested resources from missions (i.e., a set of object methods, called actions) by available resources. It is also responsible for monitoring, reserving, allocating and deallocating each resource. Given the driven situations, A QoS Management Agent controls resources when it met errors through the Resource Manager to guarantee requested QoS requirements. If there 
is some error resource due to low resource availability, it performs QoS resource error detection-recovery. This system resolves the errors by recovering resources for supporting high priority missions. To effectively identify and resolve QoS conflicts, we need to capture the relationships between mission, actions, its related QoS requirements, and resources. In this paper, the FTA access requires situation-aware fault-tolerance QoS, in which the different fault-tolerance can be automatically enforced according to different situations such as wired or wireless network environment.

\section{Conclusion}

In this paper, we proposed a QoS resource error detection-recovery model called "FTA" for situation-aware middleware. An adaptive distance education system is used as an illustrative example of the FTA model and its resource error detection-recovery. The focus of situation-aware ubiquitous computing has increased lately. An example of situation-aware applications is a multimedia education system. The development of multimedia computers and communication techniques has made it possible for a mind to be transmitted from a teacher to a student in distance environment. This paper proposed an Intelligence Fault Tolerance Agent in situation-aware middleware framework model. FTA provided several functions and features capable of developing multimedia distant education system among students and teachers during lecture. FTA is a system that is suitable for detecting and recovering software error based on distributed multimedia education environment as FTA by using software techniques. This method detects an error by using process database. The purpose of this research is to return to a healthy state or at least an acceptable state for FTA session. It is to recover application software running on situation-aware ubiquitous computing automatically. When an error occurs, FTA inspects it by using API function for process database. If an error is found, FTA decides whether it is hardware error or software error. In case of software error, it can be recoverable. FTA informs Daemon and Session Manager of the fact. As they receive the information from the FTA, Daemon and Session Manager recovers from the error. The purpose of FTA system is to maintain and recover for FTA session automatically.

In the future work, fault-tolerance system will be generalized to be used in any environment, and we will progress the study of domino effect for distributed multimedia environment as an example of situation-aware applications.

\section{References}

C. W. Loftus, E. M. Sherratt, R. J. Gautier, A. M. Grandi, D. E. Price and M. D. Tedd (1995). Distributed Software Engineering-The Practitioner Series, Prentice Hall Inc., Herfordshire, UK

Dhiraj K. Pradhan (1996). Fault-Tolerant Computer System Design, Prentice Hall Ptr

Francois Fluckiger, (1995). Understanding Networked Multimedia-Application and Technology Technology, Prentice Hall Inc., Herfordshire, UK

Gil C. Park, Dae J. Hwang (1995). Design of a multimedia distance learning system: MIDAS, Proceedings of the ISATED international conference, Pittsburgh, USA

Gordon Blair, Jean-Bernard Stefani (1997). Open Distributed Processing and Multimedia, Addison-Wesley 
Grudin, J. (1994). CSCW: History and focus, IEEE Computer 27(5), pp. 19-26

ITU-T Recommendation (1995). T.122 Multipoint Communication Service for Audiographics and Audiovisual Conferencing Service Definition, ITU-T SG8

Jae Y. Ahn, Gil C. Park and Dae J. Hwang (1996). A Dynamic Window Binding Mechanism for Seamless View Sharing in Multimedia Collaboration, Proceedings of $14^{\text {th }}$ ISATED International Conference, Innsbruck, Austria

Jae Young Ahn, Gyu mahn Lee, Gil Chul Park, Dae Joon Hwang (1996). An implementation of Multimedia Distance Education System Based on Advanced Multi-point Communication Service Infrastructure: DOORAE, In proceedings of the IASTED International Conference Parallel and Distributed Computing and Systems, October 1619, 1996, Chicago, Illinois, USA.

Jiten Rama, Judith Bishop (2006). Survey and Comparison of CSCW Groupware applications, Proceedings of SAICSIT 2006, pp. 507-512, South Africa

H. Zhang A. Kankanhalli, S. Smoliar (1995). Automatic Partitioning of Full-motion Video, A Guided Tour of Multimedia Systems and Applications, IEEE Computing Society Press

Man Seok Yang, Kim Dae Hyun, Lee Kyung Oh, Yoon Young Park (2009). A LEACH-based Clustering protocol for the Connection Persistent Improvement in Sensor Networks, Proceedings of the $8^{\text {th }}$ APIS, pp. 507-512, ISSN 1976-7587, University of the Ryukyus, Jan. 11-12, 2009, APIS, Okinawa, Japan

Michael G.Moore, Greg Kearsley (1996). DISTANCE EDUCATION A System View, An International Thomson Publishing Company

Saha, D, Mukherjee, A. (2003). Pervasive computing: a paradigm for the $21^{\text {st }}$ century, IEEE Computer, pp. 25-31, Volume: 36, Issue: 3, March 2003,

Satyanarayanan, M. (2001). Pervasive computing: vision and challenges, IEEE Personal Communications, pp. 10-17, IEEE, Volume: 8 Issue: 4, Aug. 2001

S. Yau, F. Karim, Y. Wang, B. Wang, and S. Gupta (2002). Reconfigurable Context-Sensitive Middleware for Pervasive Computing, IEEE Pervasive Computing, 1(3), pp. 33-40, July-September 2002

S. Yau and F. Karim (2002). Adaptive Middleware for Ubiquitous Computing Environments, Design and Analysis of Distributed Embedded Systems, Proc. IFIP 17th WCC, pp. 131-140, August 2002, Vol. 219.

T. Zhang and C.-C, J. Kuo (1999). Hierarchical Classification of Audio Data For Archiving and Retrieval, ICASSP'1999, Vol. 6, pp.3001-3004, Phoenix

Victor P. Nelson and Bill D. Carroll, Fault-Tolerant Computing, IEEE Computer Society, Order Number 677, Library of Congress Number 86-46205, IEEE Catalog Number EH0254-3, ISBN 0-8186-0677-0

Younglok Lee, Seungyoung Lee, Hyunghyo Lee (2006). Development of Secure Event Service for Ubiquitous Computing, Proceedings of ICIC 2006, pp. 83-94, ISBN 3540-37255-5, August, 8, 2006, LNCIS 344, Springer, Kunming, China 


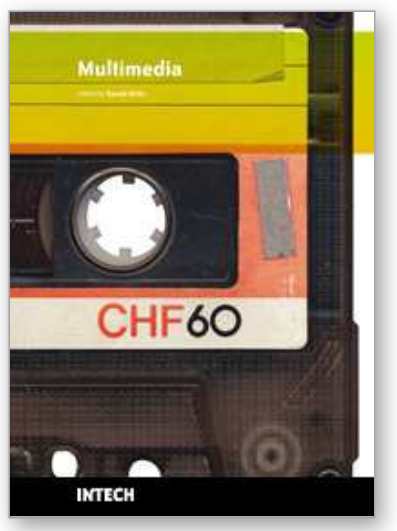

\author{
Multimedia \\ Edited by Kazuki Nishi
}

ISBN 978-953-7619-87-9

Hard cover, 452 pages

Publisher InTech

Published online 01, February, 2010

Published in print edition February, 2010

Multimedia technology will play a dominant role during the 21 st century and beyond, continuously changing the world. It has been embedded in every electronic system: PC, TV, audio, mobile phone, internet application, medical electronics, traffic control, building management, financial trading, plant monitoring and other various man-machine interfaces. It improves the user satisfaction and the operational safety. It can be said that no electronic systems will be possible without multimedia technology. The aim of the book is to present the stateof-the-art research, development, and implementations of multimedia systems, technologies, and applications. All chapters represent contributions from the top researchers in this field and will serve as a valuable tool for professionals in this interdisciplinary field.

\title{
How to reference
}

In order to correctly reference this scholarly work, feel free to copy and paste the following:

Eung-Nam Ko (2010). An Intelligence Fault Tolerance Agent for Multimedia CSCW based on SituationAwareness, Multimedia, Kazuki Nishi (Ed.), ISBN: 978-953-7619-87-9, InTech, Available from: http://www.intechopen.com/books/multimedia/an-intelligence-fault-tolerance-agent-for-multimedia-cscw-basedon-situation-awareness

\section{INTECH}

open science | open minds

\section{InTech Europe}

University Campus STeP Ri

Slavka Krautzeka 83/A

51000 Rijeka, Croatia

Phone: +385 (51) 770447

Fax: +385 (51) 686166

www.intechopen.com

\section{InTech China}

Unit 405, Office Block, Hotel Equatorial Shanghai

No.65, Yan An Road (West), Shanghai, 200040, China 中国上海市延安西路65号上海国际贵都大饭店办公楼 405 单元 Phone: +86-21-62489820

Fax: +86-21-62489821 
(C) 2010 The Author(s). Licensee IntechOpen. This chapter is distributed under the terms of the Creative Commons Attribution-NonCommercial-ShareAlike-3.0 License, which permits use, distribution and reproduction for non-commercial purposes, provided the original is properly cited and derivative works building on this content are distributed under the same license. 